\title{
Design of Experiments of AIN Reactive Sputtering
}

\author{
Pavel Mach, and Jana Kolářová \\ Department of Electrotechnology, Faculty of Electrical Engineering, \\ Czech Technical University in Prague, Prague, Czech Republic \\ mach@fel.cvut.cz
}

\begin{abstract}
Reactive sputtering has been used for fabrication of AlN films. The process has been investigated in two levels of power of a generator, in two levels of the working pressure and in two levels of the working gas flow. The AlN films have been fabricated on a bottom Al electrodes and after sputtering of the AlN film a top Al electrode has been evaporated across the bottom one. This way capacitors have been formed. The capacitance and the loss factor of these capacitors have been measured. The process of fabrication of AlN films has been mathematically described using DOE.
\end{abstract}

\section{INTRODUCTION}

AIN films have many applications in microelectronics and optics. Typical applications are barrier films, dielectric films for thin film capacitors and optical films. There are two dominating methods for fabrication of these films:

A physical vapor deposition (PVD) based on sputtering of an AlN target. Working gas used for this process is Ar.

$\rightarrow$ A plasma enhanced chemical vapor deposition (PECVD) based on sputtering of a target in a reactive gas. Aluminum target and a mixture of $\mathrm{N} 2$ and $\mathrm{Ar}$ as a working atmosphere are used for this process [1].

The PVD process is easier for control and the films are well defined. However, the target is heated on the high temperature during sputtering and is broken very often. With respect to the high price of this target, the PECVD process is preferred.

"Tuning" of working conditions of PECVD is not simple. This process depends on following factors namely:

On sputtering of the target.

$\rightarrow$ On volume plasmo-chemical processes $\left(\mathrm{Al}+\mathrm{N}_{2}+\mathrm{Ar}\right)$ and on a ratio between number of sputtered atoms of aluminum and number of molecules of the reactive gas.

On surface synthesis of an AlN film.
* Theoretical description of a PECVD process is very complicated. Therefore DOE has been used for calculation of a mathematical model, which describes this process.

\section{BASIC Methods OF AIN ThIN FILMS FABRICATION}

Physical vapor deposition is based on bombardment of an AlN target by positive ions of a working gas. This gas is inert. Atoms of AlN are sputtered from the target and create a thin AIN film on a substrate. The pressure of a working gas used for this process is from 4 to $6 \mathrm{~Pa}$ usually.

Intensity of an ion rain on the target is supported by a magnetic field usually. This field causes a spiral movement of electrons, intensifies ionization gain and bombardment of the target with positive ions of working gas.. Such an arrangement is called magnetron sputtering. The schematic arrangement of a magnetron is shown in Fig. 1.

There are two types of magnetrons used for magnetron sputtering. A planar type is shown in Fig. 2, a circle type in Fig. 3.

The principle of a process of magnetron sputtering is schematically shown in Fig. 4. 


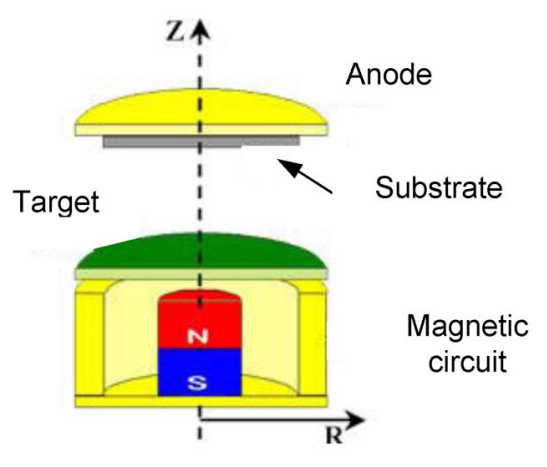

Fig. 1 Schematic arrangement of a magnetron

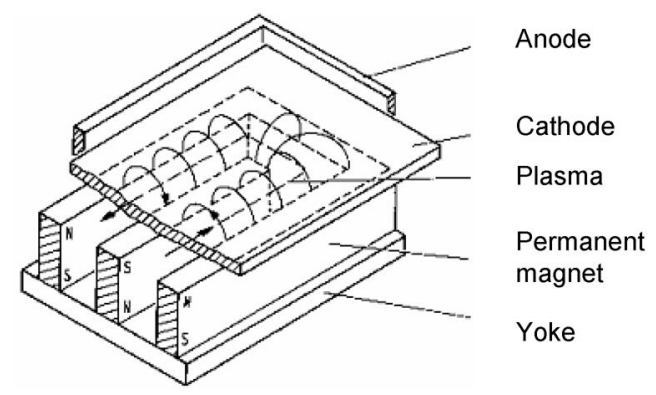

Fig. 2 Planar magnetron

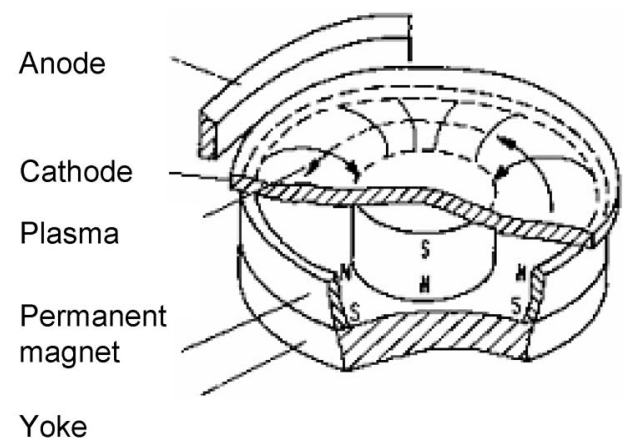

Fig. 3 Circle magnetron

A plasma enhanced chemical vapor deposition (PECVD) is based on sputtering of a target in a mixture of an inert and reactive gas. Arrangement of PECVD is the same like arrangement of PVD. However, instead inert working gas is used a mixture of inert and reactive gas. Atoms sputtered from the target react with the molecules of the reactive gas and create a new reactant, which covers surface of a substrate and create the thin film.

Basic parameters, which influence quality and grow rate of a sputtered film are as follows:

Kinetic energy of the sputtered atoms.

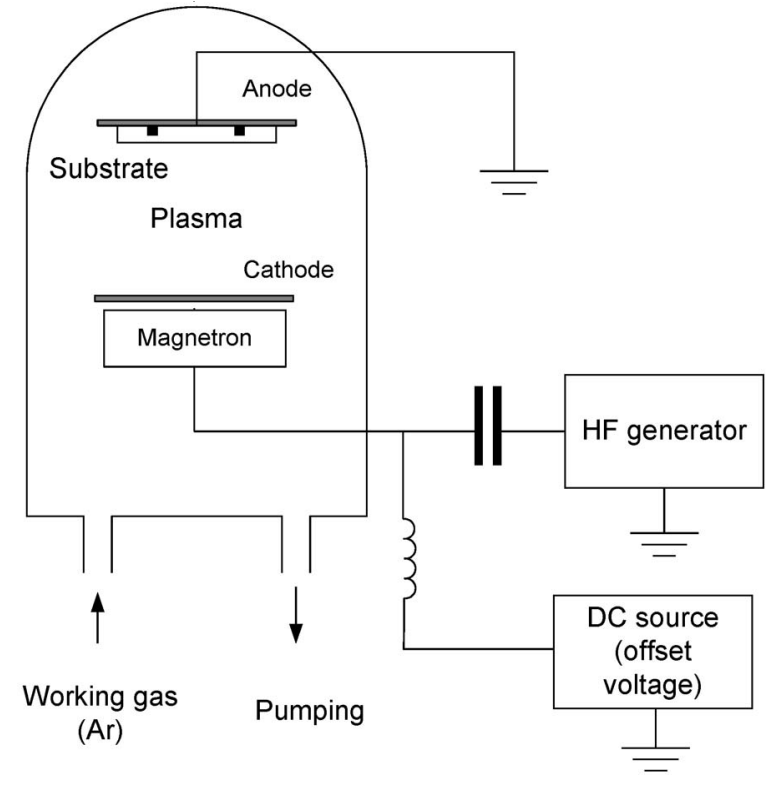

Fig. 4 Principle of HF sputtering

$\rightarrow$ DC offset of a substrate.

$\rightarrow$ Ratio between sputtered atoms of the target and number of molecules of reaction gas.

$\rightarrow$ Temperature of the target.

$\rightarrow$ Power of a magnetron.

$\rightarrow$ Ratio between the reactive gas and the inert gas in the recipient.

The theory of reactive sputtering is very complicated, because too many factors influence the result of this process. Therefore the process has been mathematically described using DOE. The calculation of a model is necessary with respect to optimization of results of the PECVD process.

\section{EXPERIMENTAL}

Quality of fabricated AIN films has been evaluated according to the properties of capacitors having AlN dielectric. Six capacitors have been formed on one glass substrate (Knittel Glasser L762601). Bottom Al electrodes of capacitors have been evaporated 
(equipment Edwards, model 19E6/425), then the AIN film has been deposited using a PECVD process in a magnetron sputtering equipment Balzers PLS 160. The process been

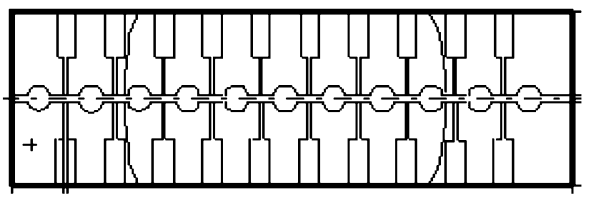

Fig. 5 Substrate with formed capacitors

finalized by evaporation of a top Al electrode across the bottom ones. The substrate with formed capacitors is shown in Fig. 5. The area of capacitors has been $0,75 \mathrm{~mm}^{2}$.

The frequency dependence of the capacitance and the loss factor of capacitors with AIN dielectric have been measured using a RLCG Meter BM595.

The process of sputtering has been investigated for the power of a generator $70 \mathrm{~W}$ and $150 \mathrm{~W}$, for the working pressure $1,5 \mathrm{~Pa}$ and 2,5 $\mathrm{Pa}$ and for the $\mathrm{N} 2$ flow $10 \mathrm{ml} / \mathrm{min}$ and $15 \mathrm{ml} / \mathrm{min}$.

DOE has been used for calculation of mathematical model of AlN films fabrication. Capacitance of the capacitors and their loss factor have been investigated.

\section{Principle of DOE}

The principle of DOE is shown in Tab. 1. A schematic diagram of DOE is presented for the simplest type of DOE, experiments of the type $2^{2}$. Schematic diagram is shown in Tab. 1. Type of experiments $2^{3}$ has been used for our calculation

Tab. 1 Schematic diagram of DOE

\begin{tabular}{|c|c|c|c|}
\hline \multicolumn{3}{|c}{ A1 } & \multicolumn{3}{c|}{ A2 } \\
B1 & B2 & B1 & B2 \\
A1B1 & A1B2 & A2B1 & A2B2 \\
$(1)$ & b & $a$ & ab \\
\hline$y 11$ & y21 & y31 & y41 \\
\hline$\cdots$ & $\cdots$ & $\cdots$ & $\cdots$ \\
\hline$\cdots$ & $\cdots$ & $\cdots$ & $\cdots$ \\
\hline$y 1 n$ & $y 2 n$ & $y 3 n$ & $y 4 n$ \\
\hline
\end{tabular}

The top part of the table (strongly boxed) represents the diagram of experiments. In the bottom part of the table measured results are presented. Every measurement has been n-times repeated.

The influence of technological factors and their interactions on the output parameter of a process has to be investigated at first. Formulas for these calculations are:

$$
\begin{aligned}
& Z_{A}=a+a c+a b+a b c-[(1)+c+b+b c \\
& Z_{B}=b+b c+a b+a b c-[(1)+c+a+a c] \\
& Z_{C}=c+b c+a c+a b c-[(1)+a+b+a b] \\
& Z_{A B}=(1)+c+a b+a b c-a-b-b c-a c \\
& Z_{A C}=(1)+b+b c+a b c-a-c-b c-a b \\
& Z_{B C}=(1)+a+b c+a b c-b-c-a c-a b \\
& Z_{A B C}=a+b+c+a b c-(1)-a b-b c-a c
\end{aligned}
$$

Total sum of squares of differences is calculated according to the formula:

$$
S_{0}=\sum_{i=1}^{d} \sum_{j=1}^{r}\left(y_{i, j}-\frac{1}{r \cdot d} \cdot \sum_{i=1}^{d} \sum_{j=1}^{r} y_{i, j}\right)^{2}
$$

Residuum sum of squares is calculated as:

$$
S_{r}=\sum_{i=1}^{d} \sum_{j=1}^{r} y_{i, j}{ }^{2}-\frac{1}{r} \sum_{i=1}^{d} R_{i}^{2}
$$

Testing characteristics: $S$

$$
F_{A}=\frac{S_{A}}{\frac{S_{r}}{v}} \quad F_{A B C}=\frac{S_{A B C}}{\frac{S_{r}}{v}}
$$

$$
\text { …...... } \quad F_{B}=\frac{S_{B}}{\frac{S r}{v}}
$$

A mathematical model can be written as:

$$
\begin{aligned}
y= & b_{0}+b_{1} x_{1}+b_{2} x_{2}+\ldots+b_{n} x_{n}+ \\
& b_{12} x_{1} x_{2}+b_{n-1, n} x_{n-1} x_{n}+\ldots \ldots . \\
& \ldots+b_{123} x_{1} x_{2} x_{3}+\ldots \ldots \ldots \ldots .
\end{aligned}
$$

Coefficients of the model can be calculated as: 
$b_{0}=\frac{1}{d} \sum_{i=1}^{d} \bar{y}_{i} \quad ; \quad b_{k}=\frac{1}{d} \sum_{i=1}^{d} x_{k, i} \bar{y}_{i}$

$k=1,2, \ldots, n$

A test characteristic of a mathematical model is:

$$
\begin{aligned}
& F=\frac{\frac{S}{d \cdot r-n-1}}{\frac{S_{r}}{v}} \text { for } d \cdot r>n+1 \\
& v=d \cdot(r-1)
\end{aligned}
$$

\section{Results}

Following mathematical models have been found: A model for the capacitance:

$C=0,970-0,0076 a+0,206 b-0,0021 c+0,00015 a c$

A model for the loss factor:

$D=-0,302+0,0023 a+0,184 b+0,0256 c-0,0012 a b--$ $0,013 b c-0,00012 a c+0,000088 a b c$

Where a ... power of the generator ( 70 to $150 \mathrm{~W}), \mathrm{b}$... Ar pressure (1,5 to $2,5 \mathrm{~Pa}), \mathrm{c} \ldots \mathrm{N} 2$ flow (10 to 15 $\mathrm{ml} / \mathrm{min}$ ).

An example of the model for calculation of $\mathrm{D}$ is shown in Fig. 6. The shape of the model is presented for two parameters only.

Both the models have been verified by comparison with parameters of capacitors fabricated in the middle and near the borders of working conditions. The inaccuracy of the models has been lower than $10 \%$.

Frequency dependence of the capacitance $\mathrm{C}$ and the loss factor D of AlN capacitors is shown in Fig. 7. The conditions of fabrication have been: power $70 \mathrm{~W}$, pressure of $\mathrm{Ar} 1,5 \mathrm{~Pa}$, flow of $\mathrm{N}_{2} 10 \mathrm{ml} / \mathrm{min}$.

\section{Conlusions}

It has been found that the fabrication of AlN using PECVD method depends strongly on the ratio between concentration of $\mathrm{Ar}$ and $\mathrm{N}_{2}$. The power of the generator influences growth rate of the layer too, however this influence is less significant than ratio of gases concentration.

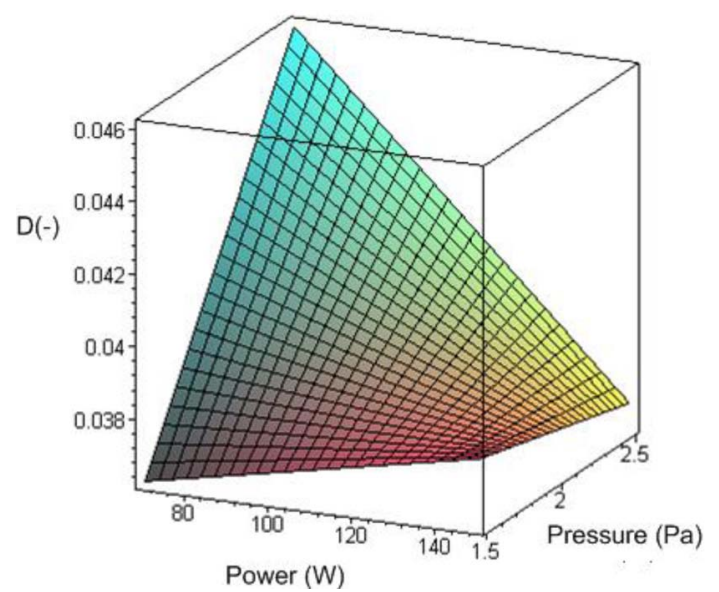

Fig. 6 Model, which has been found for calculation of D

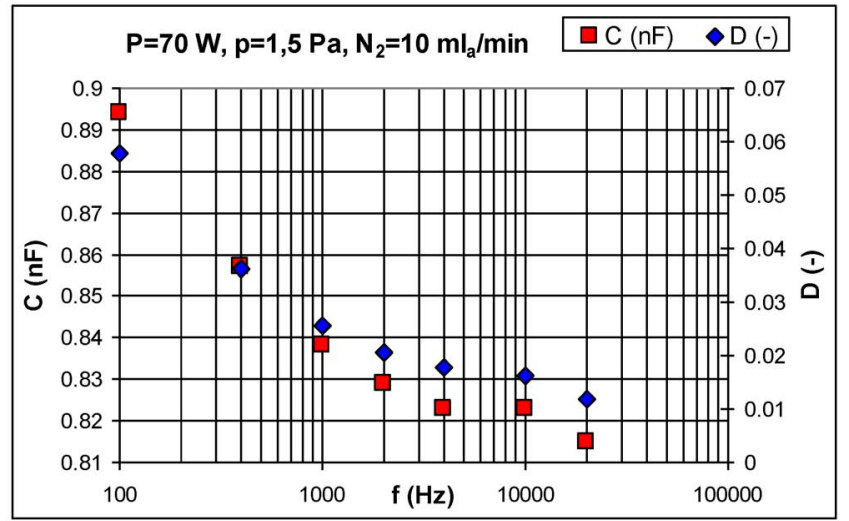

Fig. 7 Frequency dependence of capacitance of AlN capacitor

The calculated mathematical models fit the process with surprising accuracy.

\section{REFERENCES}

1. Chen, F. F., Chang, J. P.: Lecture Notes on Principles of Plasma Processing. Kluwer. 2003

\section{ACKNOWLEDGEMENT}

The work has been carried out as a part of a project "Diagnostics of Materials", number MSM6840770021 